\title{
High Resolution through Graded-Index Microoptics
}

\author{
Victor V. Kotlyar, ${ }^{1,2}$ Alexey A. Kovalev, ${ }^{1,2}$ Anton G. Nalimov, ${ }^{1,2}$ and Sergey S. Stafeev ${ }^{1,2}$ \\ ${ }^{1}$ Laser Measurements Laboratory, Image Processing Systems Institute, the Russian Academy of Sciences, \\ 151 Molodogvardeyskaya Street, Samara 443001, Russia \\ ${ }^{2}$ Technical Cybernetics Subdepartment, S. P. Korolyov Samara State Aerospace University, \\ 34 Moskovskoe Shosse, Samara 443086, Russia
}

Correspondence should be addressed to Alexey A. Kovalev, alanko@smr.ru

Received 3 April 2012; Accepted 31 May 2012

Academic Editor: Giancarlo Righini

Copyright ( 2012 Victor V. Kotlyar et al. This is an open access article distributed under the Creative Commons Attribution License, which permits unrestricted use, distribution, and reproduction in any medium, provided the original work is properly cited.

\begin{abstract}
By solving Helmholtz equations, relationships to describe propagating modes in an arbitrary graded-index planar waveguide are derived. We show that in the quadratic- and secant-index waveguides a minimal mode width is $0.4 \lambda / n$, where $\lambda$ is the wavelength in free space and $n$ is the refractive index on the fiber axis. By modeling in FullWAVE, we show that the high-resolution imaging can be achieved with half-pitch graded-index Mikaelian microlenses (ML) and Maxwell's "fisheye" lenses. It is shown that using a $2 \mathrm{D} \mathrm{ML}$, the point source can be imaged near the lens surface as a light spot with the full width at half maximum (FWHM) of $0.12 \lambda$. This value is close to the diffraction limit for silicon $(n=3.47)$ in $2 \mathrm{D}$ media FWHM $=0.44 \lambda / n=0.127 \lambda$. We also show that half-pitch $\mathrm{ML}$ is able to resolve at half-maximum two close point sources separated by a $0.3 \lambda$ distance.
\end{abstract}

\section{Introduction}

Recent advances in microoptics and nanophotonics have made possible the focusing of coherent laser light into a subwavelength spot or the superresolution imaging of a point source of light. The subwavelength focusing beyond the diffraction limit of $0.5 \lambda / n$, where $\lambda$ is the wavelength in free space and nis the material refractive index at the focus, can be performed using a superlens [1]. In 2D case, instead of conventional diffraction limit $0.5 \lambda / n$ one must use $0.44 \lambda / n$. This value can be obtained after replacing the Airy disk $2 J_{1}(x) / x$ by $\operatorname{sinc}$-function $\sin (x) / x$.

The superlens is a $2 \mathrm{D}$ planar plate made up of the metamaterial that comprises alternating metallic and dielectric layers. The electric permittivities of the layers are selected so that an effective refractive index of the composite material be equal to $n=-1$. Experiments on the superresolution through superlenses were reported in $[2,3]$. In the experiments, a superresolution of $0.4 \lambda$ was achieved [2]. A similar experiment conducted in [4] with a subwavelength silver layer operating as a superlens has shown that two lines separated by a $145 \mathrm{~nm}$ distance can be resolved when illuminated by UV light of wavelength $365 \mathrm{~nm}$, thus producing a superresolution of $0.4 \lambda$.

A far-field hyperlens reported in [5] was able to resolve two lines of width $35 \mathrm{~nm}$ spaced $150 \mathrm{~nm}$ apart for a $365 \mathrm{~nm}$ wavelength, again achieving a superresolution of $0.4 \lambda$. Note, however, that a hyperlens modelled in the form of a grating [6] was shown to achieve a superresolution of $0.05 \lambda$ at the imaging plane found $1.5 \lambda$ apart from the surface. Apparently, the absorption and scattering of light by metamaterial that occurs in real experiments was disregarded in modelling. This argument was indirectly verified by results reported in [7], in which the laser light was focused with a zone plate made up of a gold film of thickness $100 \mathrm{~nm}$. The focal spot size at half maximum was estimated to be $0.35 \lambda$, whereas the experimentally measured size of the focal spot at half maximum was found to equal $\lambda$.

Multilayer and anisotropic nanostructures allowing one to achieve a subwavelength resolution were analyzed in [810]. For example, parameters of a $1 \mathrm{D}$ eight-layer $\mathrm{Ag} / \mathrm{SiO}_{2}$ structure of thickness $400 \mathrm{~nm}$ to focus light from a $0.4 \lambda$ source into the same-size focal spot were studied in [8]. An anisotropic $2 \mathrm{D}$ nanostructure characterized by the dielectric 
permittivity tensor components $\varepsilon_{x}=0.01-i 0.01$ and $\mathcal{E}_{z}=-100$, and a $400 \mathrm{~nm}$ thickness on the $z$-axis (for $\lambda=$ $700 \mathrm{~nm}$ ) was proposed in [9]. The modelling has shown that such a structure is able to resolve two lines of width $3 \mathrm{~nm}$ placed $23 \mathrm{~nm}$ apart, providing a superresolution of $0.03 \lambda$. A nanostructure composed of two different anisotropic layers to resolve two narrow slits placed $50 \mathrm{~nm}$ apart when illuminated by a $1550 \mathrm{~nm}$ wavelength was studied in [10] by the same authors.

Candidates for achieving the superresolution can be found among the photonic crystals. Modelling conducted in [11] has shown that a $2 \mathrm{D}$ photonic-crystal slab with permittivity $\varepsilon=12$ composed of a triangular array of circular holes of radius $r=0.4 a$ ( $a$ is the hole array period) can be used as an imaging lens for wavelength $\lambda=a / 0.3$. In this case, a point source is imaged as a focal spot of size $0.3 \lambda$, whereas two point sources placed $0.5 \lambda$ apart are resolved by the $20 \%$ criterion.

In recent experiments with a $2 \mathrm{D}$ photonic-crystal slab used as a superlens, a point source of size $0.4 \lambda$ was imaged [12]. The latest publications propose an improved variant of superlenses using a nanoshell [13] or a graded-index boundary of the negative-refraction material [14]. In [15] an anisotropic layer was experimentally demonstrated to enhance and transform the evanescent surface waves into propagating light modes.

From the previous survey it follows that although theoretically superlenses allow to achieve arbitrary high resolution, experimentally the values $0.3 \lambda-0.4 \lambda$ have been obtained $[2,4,5,7,11,12]$.

Such values of resolution can be obtained with help of gradient-index optics as well. Gradient optics works as near-field optics: gradient lens is placed near the object and the image emerges in vicinity of the exit surface of the lens. Therefore, lens material affects the resolution. The limiting resolution, which can be obtained with gradient lens, decreases $n$ times in comparison with conventional refraction lens and is $0.51 \lambda / n$ in $3 \mathrm{D}$ and $0.44 \lambda / n$ in $2 \mathrm{D}$ cases. If one will use refraction lens with immersion, then index of immersion liquids does not exceed 1.5, although gradient lenses can be made of silicon with index 3.47.

In this work, we numerically demonstrate that widely known in optics graded-index Mikaelian lenses and Maxwell "fisheye" lenses may also be considered as candidates for subwavelength focusing. For a 2D Mikaelian lens, we show that a point source is imaged near the lens surface as a focal spot of size FWHM $=0.12 \lambda$ (full width at half maximum). This value is close to the diffraction limit for silicon $(n=$ 3.47 ) in $2 \mathrm{D}$ media $\mathrm{FWHM}=0.44 \lambda / n=0.127 \lambda$. This value is smaller than values earlier reported in $[7,8,11,12]$. We also show that half-pitch Mikaelian lens can resolve at halfmaximum two point sources placed $0.3 \lambda$ apart, which is smaller than reported in $[2,4,5,11]$.

Analytical relationships for modes propagating in graded-index planar waveguides were derived in $[16,17]$. A general constraint of the above works has been the assumption on the existence of one [16] or two [17] turning points of the refractive index profile of the planar waveguide. We have derived extended analytical relationships for mode

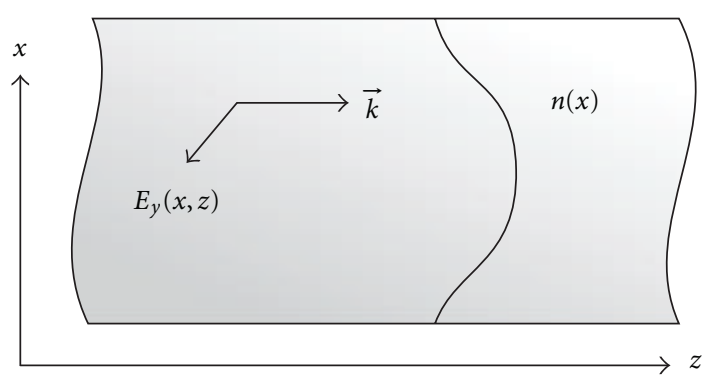

FIGURE 1: Schematic representation of the TE-wave propagating in a 2D graded-index waveguide.

solutions in the graded-index planar waveguide that have no constraints on the number of turning points of the refractive index function.

\section{Solution of the Helmholtz Equation for a 2D Graded-Index Waveguide}

Figure 1 gives a schematic representation of the problem. We consider the propagation of the TE-wave in a $2 \mathrm{D}$ gradedindex medium with the refractive index $n=n(x)$, with the electric field vector directed along the $y$-axis.

The electric field amplitude $E_{y}(x, z)$ satisfies the following Helmholtz equation [18]:

$$
\left[\frac{\partial^{2}}{\partial x^{2}}+\frac{\partial^{2}}{\partial z^{2}}+k^{2} n^{2}(x)\right] E_{y}(x, z)=0,
$$

where $k$ is the wave number. The expansion of the electromagnetic wave amplitude in terms of the transverse modes of the graded-index medium is

$$
E_{y}(x, z)=\sum_{n=0}^{\infty} C_{n}(x) \exp \left(i \beta_{n} z\right)
$$

where $\beta_{n}=k_{z n}$ is the propagation constant of the $n$th mode. From (2), it follows that the light field has an axial period $T$, so that $\beta_{n}=2 \pi n / T$. For example, in a graded-index medium with the quadratic index profile, the modes are described by the Hermite-Gauss functions that form a countable basis [19].

Substituting (2) in (1) gives an equation of the amplitude of propagating modes in the graded-index medium as

$$
\frac{d^{2} C_{n}(x)}{d x^{2}}+p_{n}(x) C_{n}(x)=0,
$$

where

$$
p_{n}(x)=k^{2} n^{2}(x)-\beta_{n}^{2}
$$

By changing variables we have

$$
C_{n}(x)=C_{n}(0) \exp \left[\int_{0}^{x} f_{n}(\xi) d \xi\right] .
$$


Equation (3) can be reduced to a nonlinear differential Whittaker equation [20] for the function $f_{n}(x)$ as follows:

$$
\frac{d f_{n}(x)}{d x}+f_{n}^{2}(x)+p_{n}(x)=0 .
$$

Equation (6) can be solved by the expansion into the Taylor series of the functions $f_{n}(x)$ and $p_{n}(x)$ as follows:

$$
f_{n}(x)=\sum_{m=0}^{\infty} C_{m}^{(n)} x^{m}, \quad p_{n}(x)=\sum_{m=0}^{\infty} p_{m}^{(n)} x^{m},
$$

where $C_{m}^{(n)}$ and $p_{m}^{(n)}$ are the unknown and known expansion coefficients of the corresponding functions. Substituting (7) into (6) yields recurrent relations $(m>0)$ for the unknown series terms in (7) as follows:

$$
C_{m}^{(n)}=-m^{-1}\left(p_{m-1}^{(n)}+\sum_{s=0}^{m-1} C_{s}^{(n)} C_{m-1-s}^{(n)}\right),
$$

where $n$ is integer and $C_{0}^{(n)}$ are indefinite constants. Then, the amplitudes of the propagating modes in a graded-index waveguide are explicitly given by

$$
C_{n}(x)=C_{n}(0) \exp \left(\sum_{m=0}^{\infty} C_{m}^{(n)} \frac{x^{m+1}}{m+1}\right)
$$

where $C_{m}^{(n)}$ are derived from the recurrent relationships (8). The coefficients $p_{m}^{(n)}$ entering in (7) are derived from

$$
\begin{aligned}
& p_{m}^{(n)}=\left.\frac{k^{2}}{m !} \frac{d^{m} n^{2}(x)}{d x^{m}}\right|_{x=0}, \quad m>0, \\
& p_{0}^{(n)}=k^{2} n^{2}(0)-\beta_{n}^{2}, \quad m=0 .
\end{aligned}
$$

Finally, the amplitude of the TE-wave propagating in the $2 \mathrm{D}$ graded-index waveguide is given by

$$
E_{y}(x, z)=\sum_{n=0}^{\infty} C_{n}(0) \exp \left[i \beta_{n} z+\sum_{m=0}^{\infty} C_{m}^{(n)} \frac{x^{m+1}}{m+1}\right] .
$$

For each mode of (11), there are two indefinite constants, $C_{n}(0)$ and $C_{0}^{(n)}$. Since the modes of (9) are neither orthogonal nor normalized, so in order for the field of (11) to be expanded in terms of the said modes (with the aim of finding the coefficients $C_{n}(0)$ ), we need to truncate both series in (11) to finite sums, then solving sets of linear algebraic equations.

The constants $C_{0}^{(n)}$ need to be selected in a special way for each mode. By way of illustration, consider one particular case. Assume the propagating modes in a quadratic-index medium: $n^{2}(x)=n_{0}^{2}-\alpha^{2} x^{2}$. In this case, $p_{n}(x)=p_{0}^{(n)}+p_{1}^{(n)} x+$ $p_{2}^{(n)} x^{2}$, where $p_{0}^{(n)}=k^{2} n_{0}^{2}-\beta_{n}^{2}, p_{1}^{(n)}=0, p_{2}^{(n)}=-k^{2} \alpha^{2}$ and $p_{m}^{(n)}=0$ at $m>2$. Let $C_{0}^{(n)}=0$, then, $C_{2}^{(n)}=-\left(C_{0}^{(n)} C_{1}^{(n)}\right)=0$ and $C_{1}^{(n)}=-p_{0}^{(n)}=\beta_{n}^{2}-k^{2} n_{0}^{2}$. For the remaining coefficients to equal zero, $C_{m}^{(n)}=0$, at $m>2$, it will suffice to put the third coefficient to equal zero: $C_{3}^{(n)}=-p_{2}^{(n)} / 3-\left(C_{1}^{(n)}\right)^{2} / 3=0$.
Whence, we obtain the following condition on the medium parameter $\alpha: k^{2} \alpha^{2}=\left(k^{2} n_{0}^{2}-\beta_{n}^{2}\right)^{2}$. Assume that $\beta_{n}=k n_{0} / \sqrt{2}$, then we obtain $\alpha=k n_{0}^{2} / 2$. Thus, we can infer that the mode of the quadratic-index waveguide, $n^{2}(x)=n_{0}^{2}\left(1-k^{2} n_{0}^{2} x^{2} / 4\right)$, is described by the Gaussian exponential function as follows:

$$
E_{y}(x, z)=C(0) \exp \left(\frac{i k n_{0} z}{\sqrt{2}}-\frac{k^{2} n_{0}^{2} x^{2}}{4}\right) .
$$

\section{General Solution for a Secant-Index Waveguide}

If there is a waveguide with the refractive index defined by a secant function on the transverse coordinate

$$
n(x)=n_{0} \operatorname{ch}^{-1}\left(\frac{k n_{0} x}{\sqrt{2}}\right),
$$

a particular solution of (1) is given by [21] as follows:

$$
E_{1 y}(x, z)=\exp \left(\frac{i k n_{0} z}{\sqrt{2}}\right) \mathrm{ch}^{-1}\left(\frac{k n_{0} x}{\sqrt{2}}\right) .
$$

In this case, the Helmholtz equation takes the following form:

$$
\left[\frac{\partial^{2}}{\partial x^{2}}+\frac{\partial^{2}}{\partial z^{2}}+k^{2} n_{0}^{2} \operatorname{ch}^{-2}\left(\frac{k n_{0} x}{\sqrt{2}}\right)\right] E_{y}(x, z)=0 .
$$
form:

We will seek the general solution of (15) in the following

$$
E_{1 y}(x, z)=A(x) \exp (i \gamma z)
$$

Substituting (16) into (15), we obtain

$$
\frac{d^{2} A(x)}{d x^{2}}+g(x) A(x)=0,
$$

where

$$
g(x)=k^{2} n_{0}^{2} \operatorname{ch}^{-2}\left(\frac{k n_{0} x}{\sqrt{2}}\right)-\gamma^{2} .
$$

It has been known [20] that (17) has a general solution

$$
A(x)=A_{1}(x)\left[C_{1}+C_{2} \int_{0}^{x} A_{1}^{-2}(\xi) d \xi\right],
$$

where $A_{1}(x)$ is a particular solution of (17), and $C_{1}, C_{2}$ are indefinite constants. In our case, the solution in (14) can be chosen as the particular solution, that is,

$$
A_{1}(x)=\operatorname{ch}^{-1}\left(\frac{k n_{0} x}{\sqrt{2}}\right), \quad \gamma=\frac{k n_{0}}{\sqrt{2}} .
$$

Then, the general solution for the secant-index waveguide is given by

$$
\begin{aligned}
E_{1 y}(x, z)= & \exp \left(\frac{i k n_{0} z}{\sqrt{2}}\right) \operatorname{ch}^{-1}\left(\frac{k n_{0} x}{\sqrt{2}}\right) \\
& \times\left\{C_{1}+\frac{C_{2}}{k n_{0} \sqrt{2}}\left[\frac{1}{2} \operatorname{sh}\left(k n_{0} x \sqrt{2}\right)+\frac{k n_{0} x}{\sqrt{2}}\right]\right\},
\end{aligned}
$$


where

$$
C_{1}=\left|E_{1 y}(x=0, z)\right|, \quad C_{2}=\left|\frac{d E_{1 y}(x=0, z)}{d x}\right| .
$$

From (14), the mode width at half maximum in the secant-index waveguide is

$$
\mathrm{FWHM}=\frac{\ln (3+2 \sqrt{2})}{\pi n_{0} \sqrt{2}} \approx \frac{0.397 \lambda}{n_{0}},
$$

where $\lambda$ is the wavelength of light in free space and $n_{0}$ is the refractive index on the waveguide axis.

\section{Partial Solution for a Quadratic-Index Waveguide}

In Section 2 of this paper, we have shown that the propagating mode of a quadratic-index waveguide with definite parameters can be described by the Gaussian exponential function (12). In this section, we shall demonstrate that this remains true of any quadratic-index medium with arbitrary parameters as follows:

$$
n^{2}(x)=n_{0}^{2}\left(1-w^{2} x^{2}\right),
$$

where $w$ is an arbitrary constant. Then, (1) reads as

$$
\left[\frac{\partial^{2}}{\partial x^{2}}+\frac{\partial^{2}}{\partial z^{2}}+k^{2} n_{0}^{2}\left(1-w^{2} x^{2}\right)\right] E_{y}(x, z)=0,
$$

and the solution of (25) will be sought for in the following form:

$$
E_{2 y}(x, z)=E_{0} \exp \left(i p z-q^{2} x^{2}\right)
$$

where

$$
q^{2}=\frac{w k n_{0}}{2}, \quad p=k n_{0} \sqrt{1-\frac{w}{k n_{0}}} .
$$

Thus, a particular mode solution of (25) is given by

$$
E_{2 y}(x, z)=E_{0} \exp \left(i k n_{0} z \sqrt{1-\frac{w}{k n_{0}}}-\frac{w k n_{0}}{2} x^{2}\right) .
$$

Note that at $w=k n_{0} / 2$ the solution in (28) coincides with that in (12). At $w=k n_{0} / 2$, it follows from (28) that the Gaussian mode width (diameter) at half maximum is

$$
\mathrm{FWHM}=\frac{\sqrt{\ln 4} \lambda}{\pi n_{0}} \approx \frac{0.38 \lambda}{n_{0}} .
$$

Comparison of (29) and (23) suggests that the both modes (the secant and the Gaussian mode) have nearly the same width. The effective width of the quadratic-index waveguide is derived from the condition $n\left(x_{0}\right)=1$, being given by

$$
2 x_{0}=\frac{2 \sqrt{n_{0}^{2}-1}}{\pi n_{0}} \lambda .
$$

At $n_{0}=1.5$, from (30), we find that $2 x_{0} \approx 0.48 \lambda$. Thus, the effective width of a glass quadratic-index planar waveguide that can only propagate the Gaussian mode of (28) equals nearly half the free-space wavelength. Note that presently such half-wave waveguides are actively used in applications [22, 23].

\section{Modeling the Propagation of Light through Graded-Index Microoptics}

First experiments on superresolution imaging with superlenses in the optical regime were reported several years ago $[3,5]$. Thus, a superresolution of $0.4 \lambda$ has been achieved in the experiment reported in $[3,5]$.

Theoretically (disregarding the absorption of the material), any degree of superresolution can be achieved with a superlens. In [9], it was shown by modeling that a hyperlens that would form a magnified subwavelength image in the near field can be implemented as a plane-parallel layer. A $400 \mathrm{~nm}$ thick anisotropic slab with dielectric permittivities $\varepsilon_{x}=0.01-i 0.01$ and $\varepsilon_{z}=-100$ was shown to resolve two $3 \mathrm{~nm}$ slits (directed along the $y$-axis) spaced $23 \mathrm{~nm}$ apart in a metallic screen with dielectric permittivity $\varepsilon=$ $1-i 10^{4}$, illuminated by a $700 \mathrm{~nm}$ TM wave. The resulting superresolution achieved is $0.05 \lambda$.

In the following subsections, using a well-known FDTD method, implemented in FullWAVE, we numerically show that the high resolution is also achievable with the aid of $2 \mathrm{D}$ graded-index microoptics.

5.1. High Resolution Imaging Using a Mikaelian Microlens $(M L)$. Because of diffraction in uniform space, two nearby point sources of light cease to be resolved at a distance much smaller than the wavelength of incident light. By way of illustration, Figure 2(a) depicts profiles of five original coherent Gaussian light sources of width $\lambda / 200$, spaced $\lambda / 50$ apart in the plane $z=5 \mathrm{~nm}$. Shown in Figure $2(\mathrm{~b})$ is the intensity profile of these sources obtained in the image plane $z=30 \mathrm{~nm}$ for the wavelength $\lambda=1550 \mathrm{~nm}$. Figure 2 suggests that two nearby light sources cease to be resolved at a distance $z$ approximately equal to the spacing between them $(z=$ $\lambda / 50)$.

Shown in Figure 3(a) is a numerically simulated image of two $35 \mathrm{~nm}$ sources spaced $150 \mathrm{~nm}$ apart obtained with a halfpitch ML [24-26]. The refractive index of this 2D secantgraded microlens is

$$
n(x)=n_{0} \operatorname{ch}^{-1}\left(\frac{\pi x}{2 L}\right)
$$

where $2 L$ is the lens length. The lens width $2 R$ is found from the condition $n(R)=1$. The lens in Figure $3(\mathrm{a})$ has the axial refractive index $n_{0}=2.1$, width $2 R=1 \mu \mathrm{m}$, length $2 L=$ $1,144 \mu \mathrm{m}$, and operating at wavelength $\lambda=365 \mathrm{~nm}$. These parameters are similar to those described in the experiment in [5].

The distance between these sources is $0.41 \lambda$, and they cannot be resolved by refraction lens. But gradient-index lens (31), which is placed close $(20 \mathrm{~nm})$ to sources, works as immersion and allows to increase resolution $n_{0}$ times, that is, to resolve the two sources separated by distance $0.44 \lambda / n_{0}$.

Figure 3(a) shows the instantaneous distribution of the electric field amplitude for the TE wave (propagating from the bottom upwards). The sources are seen to be imaged on the opposite side of the lens. Figure 3(b) shows a timeaveraged intensity profile of the electric field, $I\left(x, z=z_{0}\right)=$ $\left|E_{y}\left(x, z=z_{0}\right)\right|^{2}$, directly on the lens "rear" side, that is, 


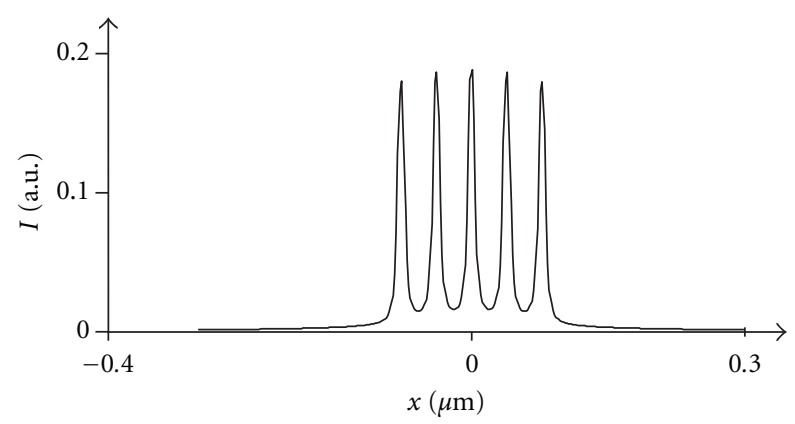

(a)

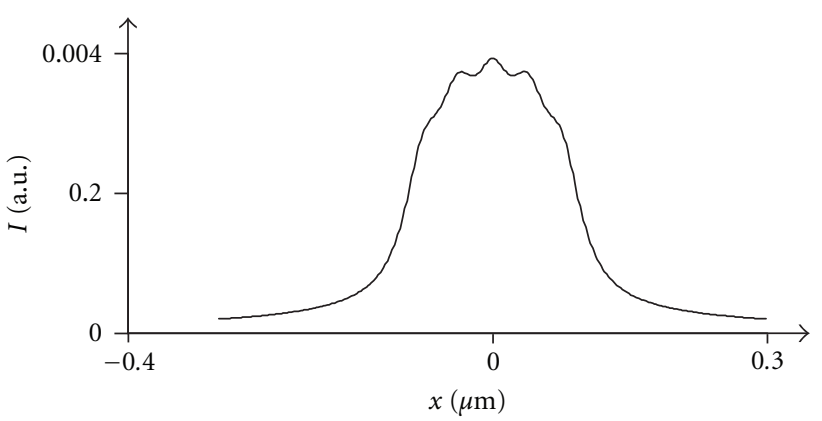

(b)

FIGURE 2: Light intensity profile near five Gaussian light sources of width $\lambda / 200$, spaced $\lambda / 50$ apart, at different distances: (a) $z=5 \mathrm{~nm}$, (b) $z=30 \mathrm{~nm}$ for $\lambda=1550 \mathrm{~nm}$ (intensity is plotted in relative units).

at a distance of $z_{0}=2 L$ from the lens "front" side. In the experiment, the sources under imaging were put $20 \mathrm{~nm}$ before the lens "front" side.

From Figure 3(b), it is seen that two sources spaced $150 \mathrm{~nm}$ apart (with center-to-center distance of $180 \mathrm{~nm}$ ) can be confidently resolved. The high resolution achieved in Figure $3(\mathrm{~b})$ is $0.41 \lambda$. From Figure $3(\mathrm{~b})$, it can also be found that the point source imaged with the half-pitch ML has a width at half maximum of FWHM $=100 \mathrm{~nm}=0.27 \lambda$. This value is comparable with the diffraction limit FWHM = $0.44 \lambda / n=0.21 \lambda$ in $2 \mathrm{D}$ media with index $n=2.1$. This value of high resolution, $0.41 \lambda$, is very close to that reported in $[2,3,5](0.40 \lambda)$. Note that the source image width of $0.27 \lambda$ well agrees with the minimal width of the propagating mode in a secant-graded waveguide: $0.4 \lambda / n_{0}=0.27 \lambda$ at $n_{0}=1.5$ (see (23)).

Numerical aperture of the ML is NA $=\left(n_{0}^{2}-1\right)^{1 / 2} / n_{0}=$ 0.88 for $n_{0}=2.1$. Therefore, for such lens the focal spot width at half-maximum intensity is FWHM = $0.44 \lambda /\left(n_{0} \mathrm{NA}\right)=0.24 \lambda$. This value is slightly less than obtained by simulation: FWHM $=0.27 \lambda$.

To improve the resolution of half-pitch ML, as suggested by (23) and (29), the axial refractive index was changed to $n_{0}=3.47(\mathrm{Si})$. The other simulation parameters were also changed (Figure 4(a)) to wavelength $\lambda=1 \mu \mathrm{m}$, lens width $2 R=6 \mu \mathrm{m}$, and lens length $2 L=4.92 \mu \mathrm{m}$. The simulation step along the spatial axes in all examples considered was $\lambda / 100$. The width of the Gaussian source at the original

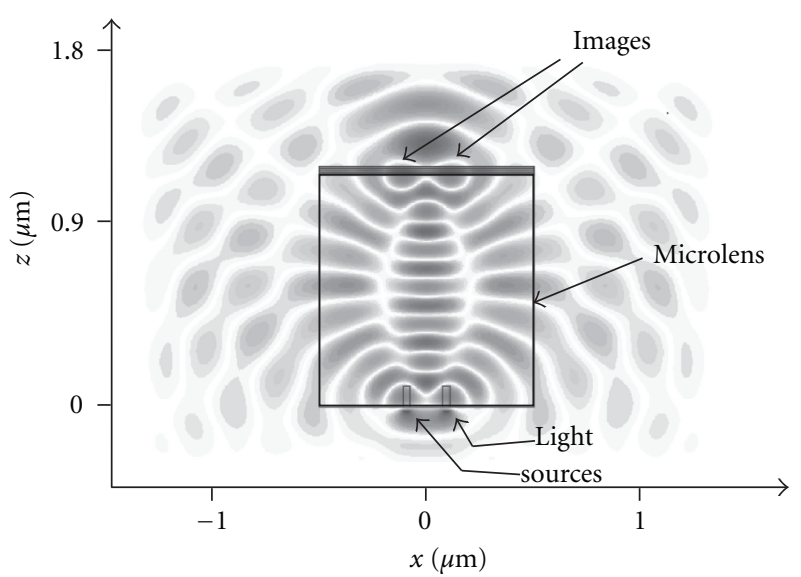

(a) .

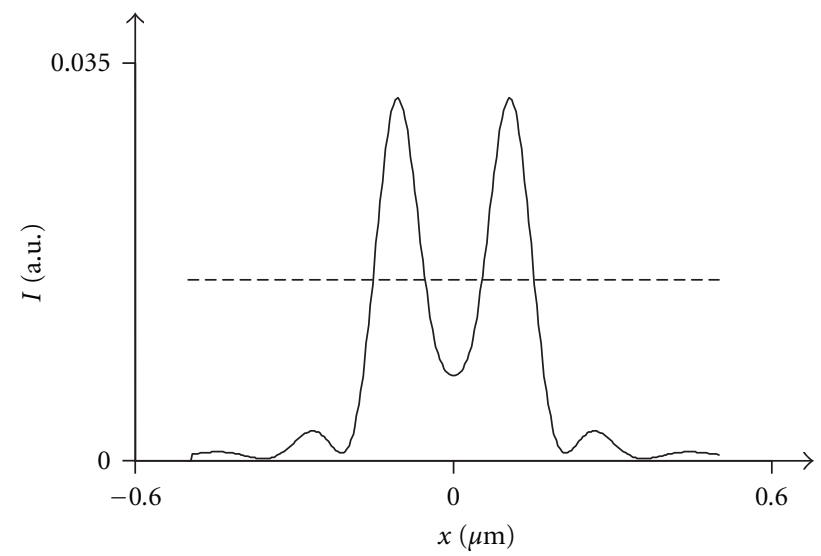

(b)

Figure 3: (a) The snapshot of the amplitude $E_{y}(x, z)$ in the ML. (b) Averaged E-vector intensities of the TE wave calculated directly on the lens "rear" side of the ML (the horizontal line indicates the half-maximum intensity plotted in relative units).

plane was $\lambda / 20$. Shown in Figure 4(a) is an instantaneous pattern of the E-vector of the light wave in the half-pitch ML calculated for the instance when the wave has travelled a $220 \mu \mathrm{m}$ distance from the source. Figure 4(b) depicts an averaged intensity of the TE-wave at the ML output plane (Figure 4(a)). The computation has shown that the intensity of Figure 4(b) corresponds to the source image width at half maximum of FWHM $=0.12 \lambda$. However, the intensity (or light power density) provides no information as to the proportion of source power propagating along the $z$-axis. This information can be derived from the projection of Poynting vector onto the optical axis, calculated at the ML output (Figure 4(a)), with the point source found at the lens input, as shown in Figure 5.

The central-maximum width of the flow of energy propagating along the $z$-axis in Figure 5 is equal to that in Figure $4(\mathrm{~b})$, being equal to FWHM $=0.12 \lambda$. The value of the diffraction limit (in 2D case) that can be achieved when focusing light in the homogeneous medium is known to be equal to $0.44 \lambda / n$, where $n$ is the refractive index of the homogeneous medium. In our case, $n_{0}=3.47$, therefore 


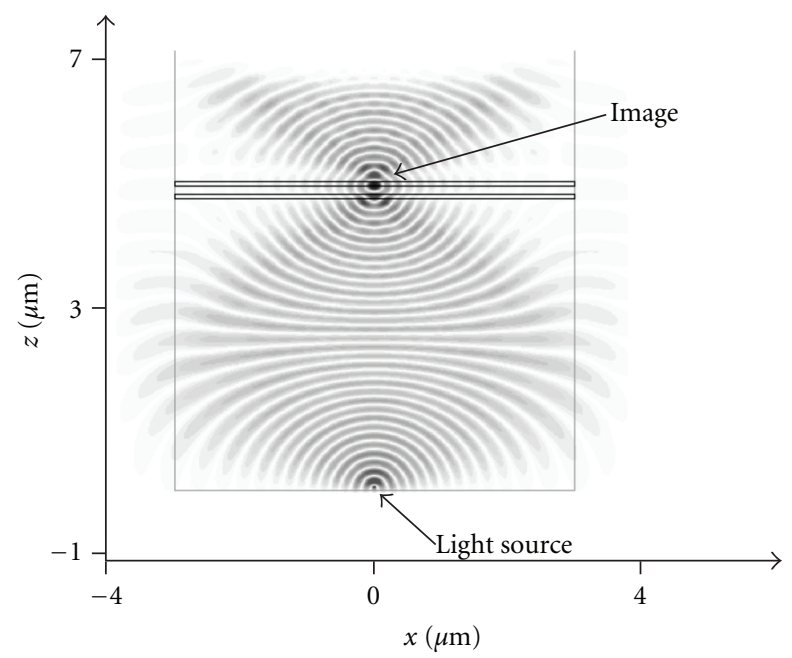

(a)

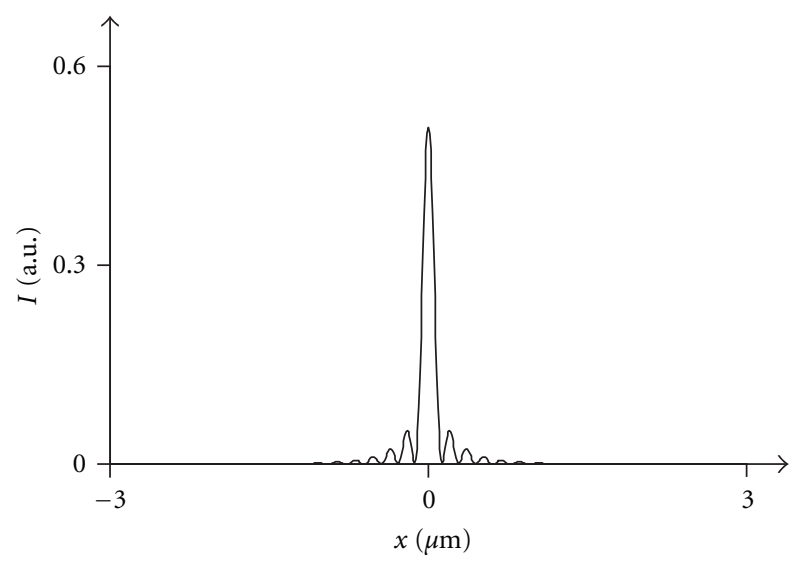

(b)

FIgURE 4: (a) Instantaneous pattern of the E-vector of the TE wave in the half-pitch ML from a point source found at the front plane (b). An averaged intensity pattern in the lens rear plane (with arbitrary units plotted on the $y$-axis).

the diffraction limit is FWHM $=0.127 \lambda$. Numerical aperture for the silicon $\left(n_{0}=3.47\right) \mathrm{ML}$ is NA $=\left(n_{0}^{2}-1\right)^{1 / 2} / n_{0}=0.96$. Therefore, the focal spot width at half maximum of intensity is FWHM $=0.44 \lambda /\left(n_{0} \mathrm{NA}\right)=0.132 \lambda$ for such lens. This value is greater than obtained by simulation FWHM $=0.12 \lambda$.

Presumably, this is because when focusing light at the two-medium interface, there is also a contribution to the focus from inhomogeneous surface waves that form interference and diffraction patterns with deep subwavelength features $[9,10]$. Figure 5 displays the fact that the surface wave play a part when imaging the point source. In a certain region on the $x$-axis, the $z$-projection of Poynting vector is negative, which means that near the output surface of ML there are both outgoing and incoming light waves, with a surface wave propagating along the microlens surface. As light propagates further in free space from the lens output surface, the light spot size quickly increases, becoming equal to the diffraction limit of $0.5 \lambda$ at a wavelength distance from the surface.

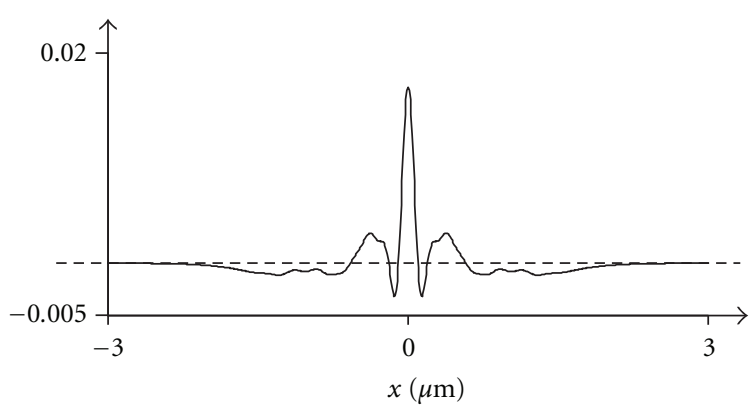

Figure 5: Profile of the $z$-projection of Poynting vector onto the $x$ axis calculated at the ML output (Figure 4(a)) with the point source at the input (in relative units).

Notice that the change of the sign of the Poynting vector projection similar to that shown in Figure 5 was earlier reported in [8], being termed as the optical vortex and interpreted as resulting from the interference between the propagating wave and the enhanced surface plasmon. Note, however, that [8] handled a 1D multilayered structure (a 1D photonic crystal).

Figure 6(a) shows the instantaneous distribution of the E-vector of the light wave in the Mikaelian lens (for the same parameters as in Figure 4(a)) illuminated by two $50 \mathrm{~nm}$ sources with center-to-center distance $300 \mathrm{~nm}$, put $10 \mathrm{~nm}$ away from the lens bottom plane. Shown in Figure 6(b) is the relative time-averaged distribution of the $z$-projection of the Poynting vector calculated at the $10 \mathrm{~nm}$ distance from the microlens output surface. It can be seen from Figure 6(b) that the resulting superresolution value is $0.3 \lambda$, which is smaller than that reported in $[2,4,5,11]$. Although point source image has width FWHM $=0.12 \lambda$ (Figures 5 and $4(\mathrm{~b}))$, only two point sources, separated by $0.3 \lambda$, are surely resolved (Figure 6(b)). This is because images of point sources interfere with each other.

Let us note that such gradient-index lens can be fabricated as a photonic-crystal lens [27].

5.2. High Resolution through a "Fisheye" Microlens. Alongside the ML discussed above, the high resolution can be achieved with other graded-index imaging optical elements for which the refractive index as a function of coordinates has been derived in an explicit analytical form. The possibility of obtaining ideal image of the point source with help of Maxwell's "fisheye" lens is justified in [28]. One such optical element is represented by Maxwell's "fisheye" [18], whose 2D refractive index is given in polar coordinates as

$$
n(r)=n_{0}\left[1+\left(\frac{r}{R}\right)^{2}\right]^{-1}
$$

where $n_{0}$ is the refractive index at the circle center and $R$ is the element radius. According to (32), the refractive index is halved at $r=R$. A disadvantage of this element is that the refractive index center-to-edge contrast cannot be larger than two, whereas in the ML the refractive index contrast 


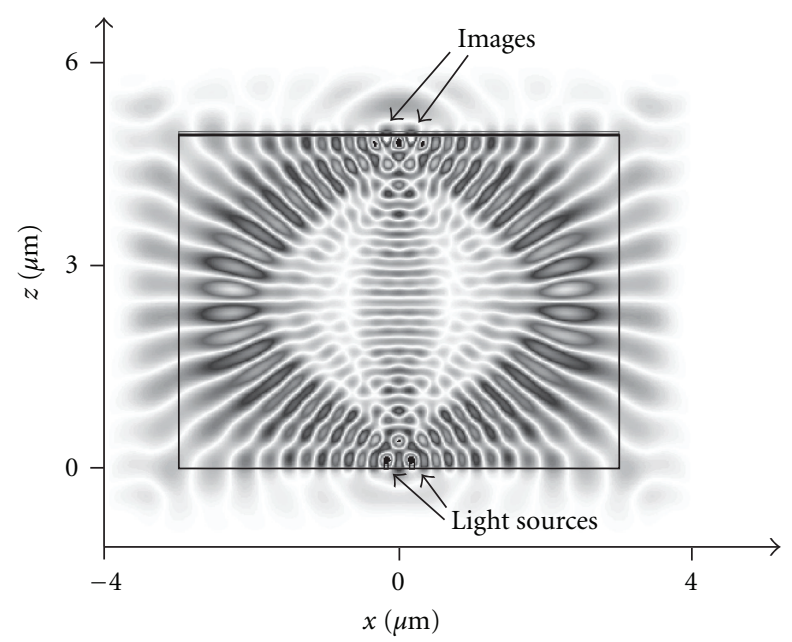

(a)

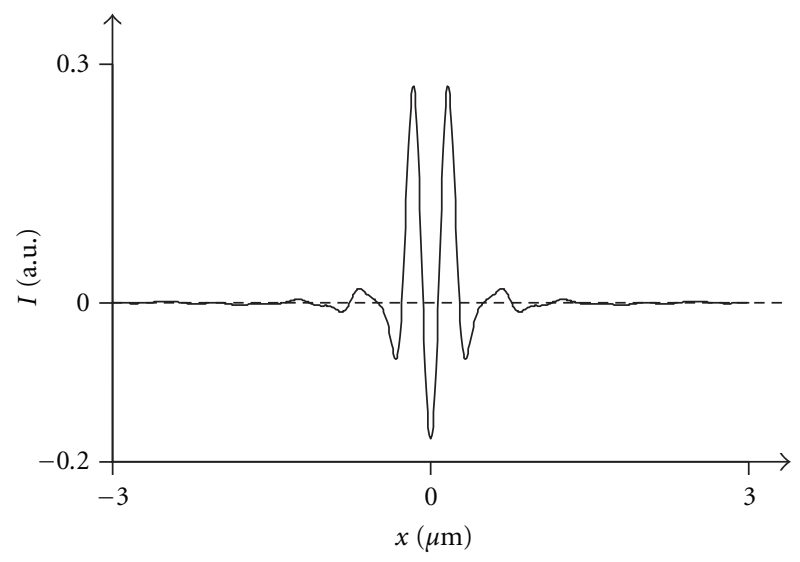

(b)

Figure 6: (a) Instantaneous distribution of the E-vector of the TE wave in the half-pitch ML of Figure 4(a) illuminated by two $50 \mathrm{~nm}$ sources with center-to-center distance $300 \mathrm{~nm}$, put $10 \mathrm{~nm}$ away from the input plane (bottom horizontal line). (b) Relative time-averaged distribution of the $z$-projection of Poynting vector calculated at $10 \mathrm{~nm}$ distance from the output plane (top horizontal line).

is a function of the material, for example, for Si ranging from 3.47 to 1 . However, with the fisheye having a circular symmetry, any source found on its surface will be perfectly imaged at the diametrically opposite point of its surface.

Figure 7(a) shows the profile of the E-vector of the TE wave in the $2 \mathrm{D}$ fisheye microlens that has two nearby point sources on its surface. The simulation was performed for the refractive index at the lens center $n_{0}=3.47$, lens radius $R=2.5 \mu \mathrm{m}$, wavelength $\lambda=1 \mu \mathrm{m}$, and two $0.05 \lambda$ sources separated by a $440 \mathrm{~nm}$ distance, which is $0.44 \lambda$. Shown in Figure $7(\mathrm{~b})$ is a time-averaged intensity pattern in the image plane (top horizontal line in Figure $7(\mathrm{a})$ ). The two sources are seen to be resolved (the resolution being $0.44 \lambda$ by the $20 \%$ Rayleigh criterion). Thus, the lens superresolution of $0.44 \lambda$ is insignificantly beyond the diffraction limit of $0.5 \lambda$.

When the microlens of Figure $7(\mathrm{a})$ is illuminated by a solitary source, the resulting intensity distribution is shown

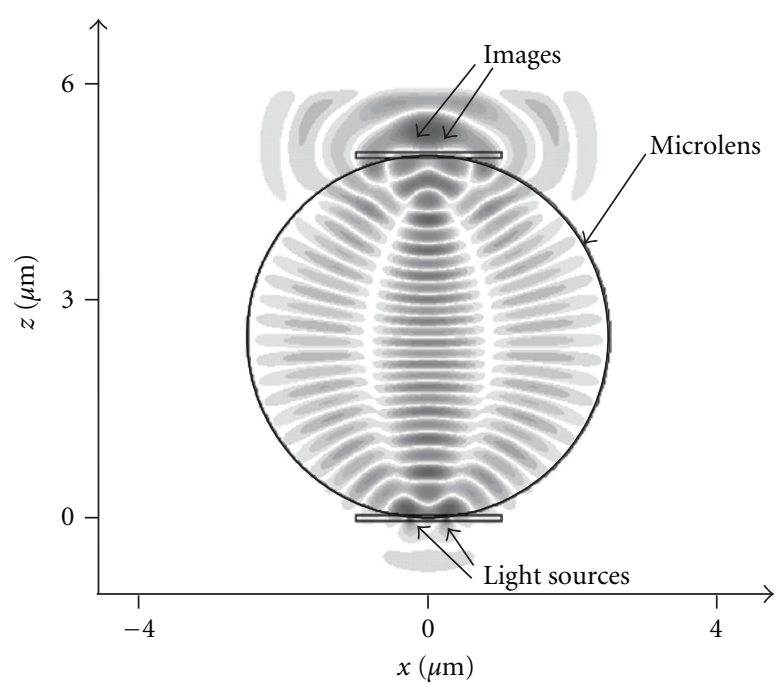

(a)

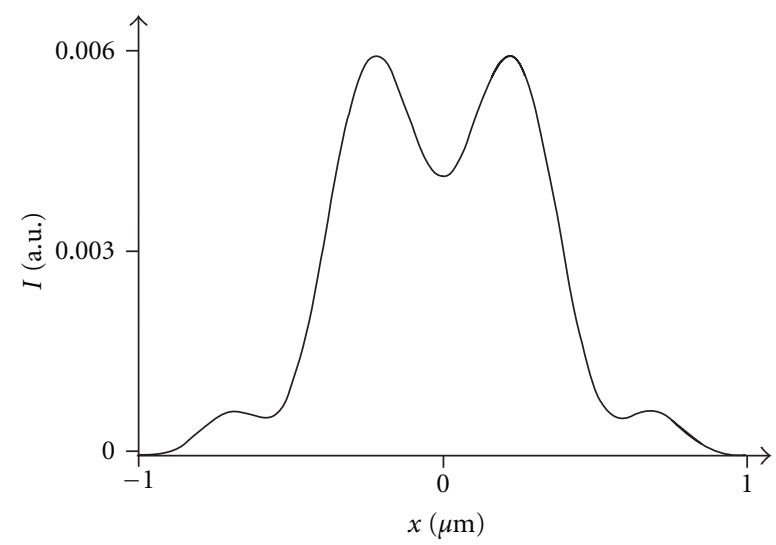

(b)

Figure 7: (a) An instantaneous distribution of the E-vector of the TE wave in the 2D fisheye microlens simulated in FullWAVE, when illuminated from bottom by two point sources (b). Relative timeaveraged intensity distribution in the image plane.

in Figure 8. The image size at half maximum is FWHM = $0.3 \lambda$. From the comparison of Figures 8 and $4(\mathrm{~b})$, we can infer that the fisheye forms a (about two-times) wider image of the point source when compared with the ML at similar parameters. Note that the magnitudes of high resolution obtained, $0.4 \lambda$ (Figure $7(\mathrm{~b})$ ) and $0.3 \lambda$ (Figure 8 ), are comparable with those reported in $[2,4,5,8,11,12]$.

Although point source image has width FWHM $=0.3 \lambda$ (Figure 8), two point sources must be separated by $0.4 \lambda$ (Figure 7(b)) distance in order to resolve them by Rayleigh criterion. This is because images of point sources interfere with each other.

\section{Conclusion}

Thus, in this work we have

(i) derived mode solutions of the Helmholtz equation for an arbitrary graded-index planar waveguide, with 


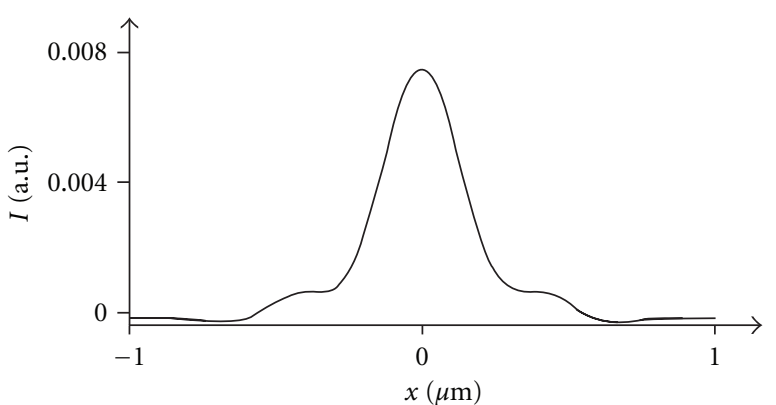

FIGURE 8: Relative time-averaged intensity pattern of the electric field in the image plane of the eyefish microlens of Figure $7(a)$ when illuminated by a single point source found on its surface.

the mode amplitude represented as the exponential of the Taylor series whose coefficients are deduced from the recurrent relations ((8), (9), and (11));

(ii) shown that the minimal mode width in quadraticand secant-index planar waveguides amounts to 0.4 of the wavelength in free space divided by the refractive index on the waveguide axis ((23) and (29));

(iii) shown by modeling in FullWAVE that graded-index half-pitch ML and fisheye lenses are capable of highresolution imaging (Figures 6 and 7);

(iv) shown that a point source is imaged through the 2D half-pitch ML as a near-surface light spot of size FWHM $=0.12 \lambda$ ( $\lambda$ is wavelength in free space), which is close to the diffraction limit for silicon $(n=$ 3.47) in $2 \mathrm{D}$ media FWHM $=0.44 \lambda / n=0.127 \lambda$ and smaller than values reported in $[8,11,12]$ (Figures 4 and 5);

(v) also shown that the half-pitch ML is able to resolve at half-maximum two nearby sources placed $0.3 \lambda$ apart, which is beyond the diffraction limit in free space of $0.44 \lambda$ for $2 \mathrm{D}$ case and smaller than values reported in $[2,4,5,11]$ (Figure 6).

\section{Acknowledgments}

The work was financially supported by the Federal Program "Research and Educational Staff of Innovation Russia" (State Contract no. 14.740.11.0016), Russian Federation Presidential grants for Support of Leading Scientific Schools (NSh4128.2012.9), a Young Researcher's grant (MK-3912.2012.2), and by the Russian Foundation for Basic Research Grant no. 12-07-00269.

\section{References}

[1] J. B. Pendry, "Negative refraction makes a perfect lens," Physical Review Letters, vol. 85, no. 18, pp. 3966-3969, 2000.

[2] R. J. Blaikie and D. O. S. Melville, "Imaging through planar silver lenses in the optical near field," Journal of Optics A, vol. 7, no. 2, pp. S176-S183, 2005.
[3] D. O. S. Melville and R. J. Blaikie, "Super-resolution imaging through a planar silver layer," Optics Express, vol. 13, no. 6, pp. 2127-2134, 2005.

[4] N. Fang, H. Lee, C. Sun, and X. Zhang, "Sub-diffractionlimited optical imaging with a silver superlens," Science, vol. 308, no. 5721, pp. 534-537, 2005.

[5] Z. Liu, H. Lee, Y. Xiong, C. Sun, and X. Zhang, "Far-field optical hyperlens magnifying sub-diffraction-limited objects," Science, vol. 315, no. 5819, p. 1686, 2007.

[6] S. Thongrattanasiri and V. A. Podolskiy, "Hypergratings: nanophotonics in planar anisotropic metamaterials," Optics Letters, vol. 34, no. 7, pp. 890-892, 2009.

[7] Y. Fu, R. G. Mote, Q. Wang, and W. Zhou, "Experimental study of plasmonic structures with variant periods for subwavelength focusing: analyses of characterization errors," Journal of Modern Optics, vol. 56, no. 14, pp. 1550-1556, 2009.

[8] K. J. Webb and M. Yang, "Subwavelength imaging with a multilayer silver film structure," Optics Letters, vol. 31, no. 14, pp. 2130-2132, 2006.

[9] H. Liu, Shivanand, and K. J. Webb, "Subwavelength imaging opportunities with planar uniaxial anisotropic lenses," Optics Letters, vol. 33, no. 21, pp. 2568-2570, 2008.

[10] H. Liu, Shivanand, and K. J. Webb, "Subwavelength imaging with nonmagnetic anisotropic bilayers," Optics Letters, vol. 34, no. 14, pp. 2243-2245, 2009.

[11] X. Wang, Z. F. Ren, and K. Kempa, "Unrestricted superlensing in a triangular two-dimensional photonic crystal," Optics Express, vol. 12, no. 13, pp. 2919-2924, 2004.

[12] B. D. F. Casse, W. T. Lu, R. K. Banyal et al., "Imaging with subwavelength resolution by a generalized superlens at infrared wavelengths," Optics Letters, vol. 34, no. 13, pp. 19941996, 2009.

[13] I. Tsukerman, "Superfocusing by nanoshells," Optics Letters, vol. 34, no. 7, pp. 1057-1059, 2009.

[14] P. C. Ingrey, K. I. Hopcraft, O. French, and E. Jakeman, "Perfect lens with not so perfect boundaries," Optics Letters, vol. 34, no. 7, pp. 1015-1017, 2009.

[15] E. A. Ray, M. J. Hampton, and R. Lopez, "Simple demonstration of visible evanescent-wave enhancement with far-field detection," Optics Letters, vol. 34, no. 13, pp. 2048-2050, 2009.

[16] Z. Cao, Y. Jiang, Q. Shen, X. Dou, and Y. Chen, "Exact analytical method for planar optical waveguides with arbitrary index profile," Journal of the Optical Society of America A, vol. 16, no. 9, pp. 2209-2212, 1999.

[17] M. S. Chung and C. M. Kim, "General eigenvalue equations for optical planar waveguides with arbitrarily graded-index profiles," Journal of Lightwave Technology, vol. 18, no. 6, pp. 878-885, 2000.

[18] M. Born and E. Wolf, Principles of Optics, Pergamon Press, Oxford, UK, 1968.

[19] W. Miller, Symmetry and Separation of Variables, AddisonWesley, Reading, Mass, USA, 1977.

[20] G. Korn and T. Korn, Mathematical Handbook, MGraw-Hill, New York, NY, USA, 1963.

[21] Y. R. Triandaphilov and V. V. Kotlyar, "Photonic crystal mikaelian lens," Optical Memory and Neural Networks, vol. 17, pp. 1-7, 2008.

[22] J. J. He and D. Liu, "Wavelength switchable semiconductor laser using half-wave V-coupled cavities," Optics Express, vol. 16, no. 6, pp. 3896-3911, 2008.

[23] X. Lin, D. Liu, and J. He, "Design and analysis of $2 \times 2$ halfwave waveguide couplers," Applied Optics, vol. 48, pp. F18F23, 2009. 
[24] A. L. Mikaelian, "Application of stratified medium for waves focusing," Doklady Akademii Nauk SSSR, vol. 81, pp. 569-571, 1951 (Russian).

[25] G. Beliakov and D. Y. C. Chan, "Analysis of inhomogeneous optical systems by the use of ray tracing. I. Planar systems," Applied Optics, vol. 36, no. 22, pp. 5303-5309, 1997.

[26] A. L. Mikaelian, "Self-focusing media with variable index of refraction," Progress in Optics, vol. 17, pp. 279-345, 1980.

[27] M. I. Kotlyar, Y. R. Triandaphilov, A. A. Kovalev, V. A. Soifer, M. V. Kotlyar, and L. O'faolain, "Photonic crystal lens for coupling two waveguides," Applied Optics, vol. 48, no. 19, pp. 3722-3730, 2009.

[28] O. Leonhard, "Perfect imaging without negative refraction," New Journal of Physics, vol. 11, Article ID 093040, 2003. 

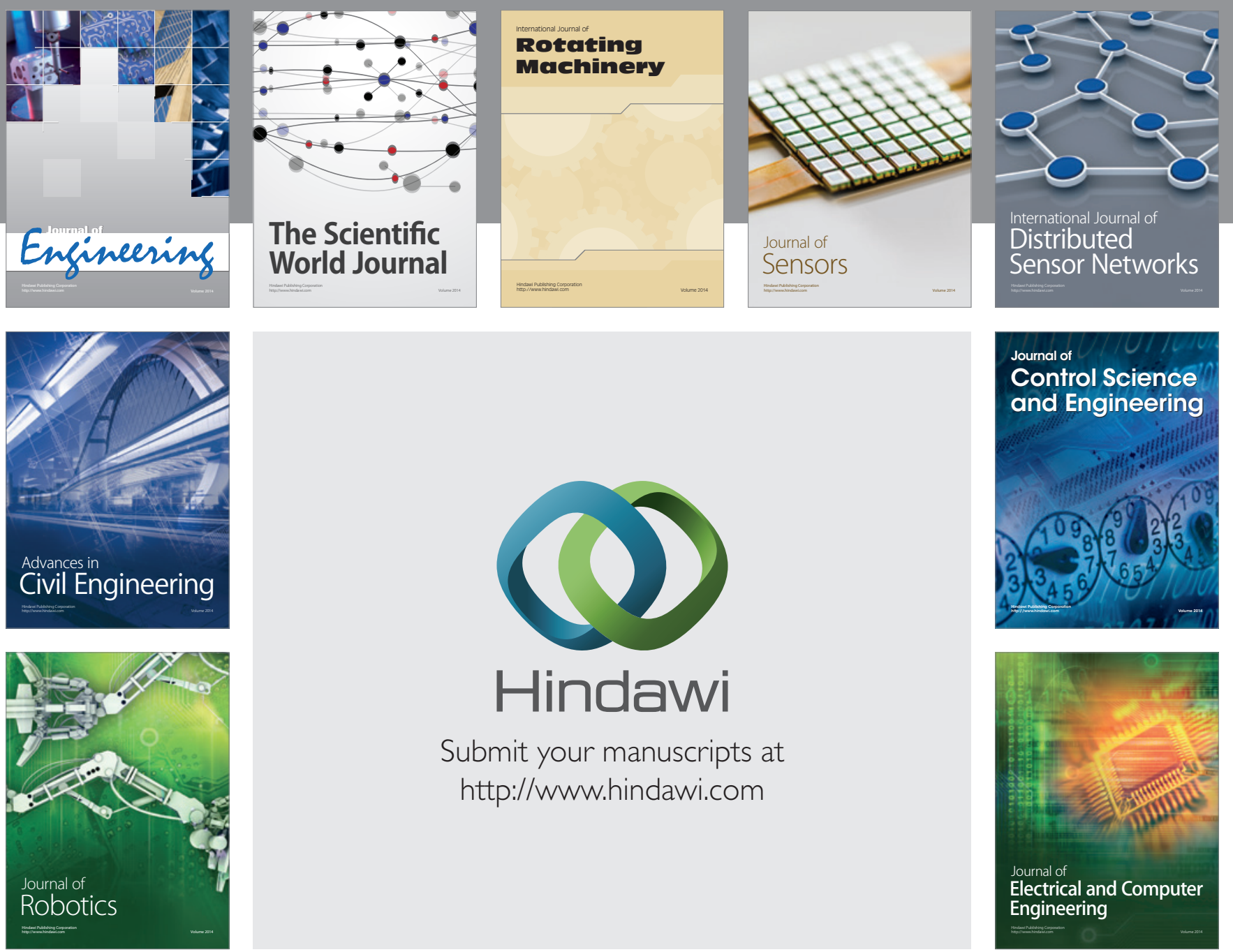

Submit your manuscripts at

http://www.hindawi.com
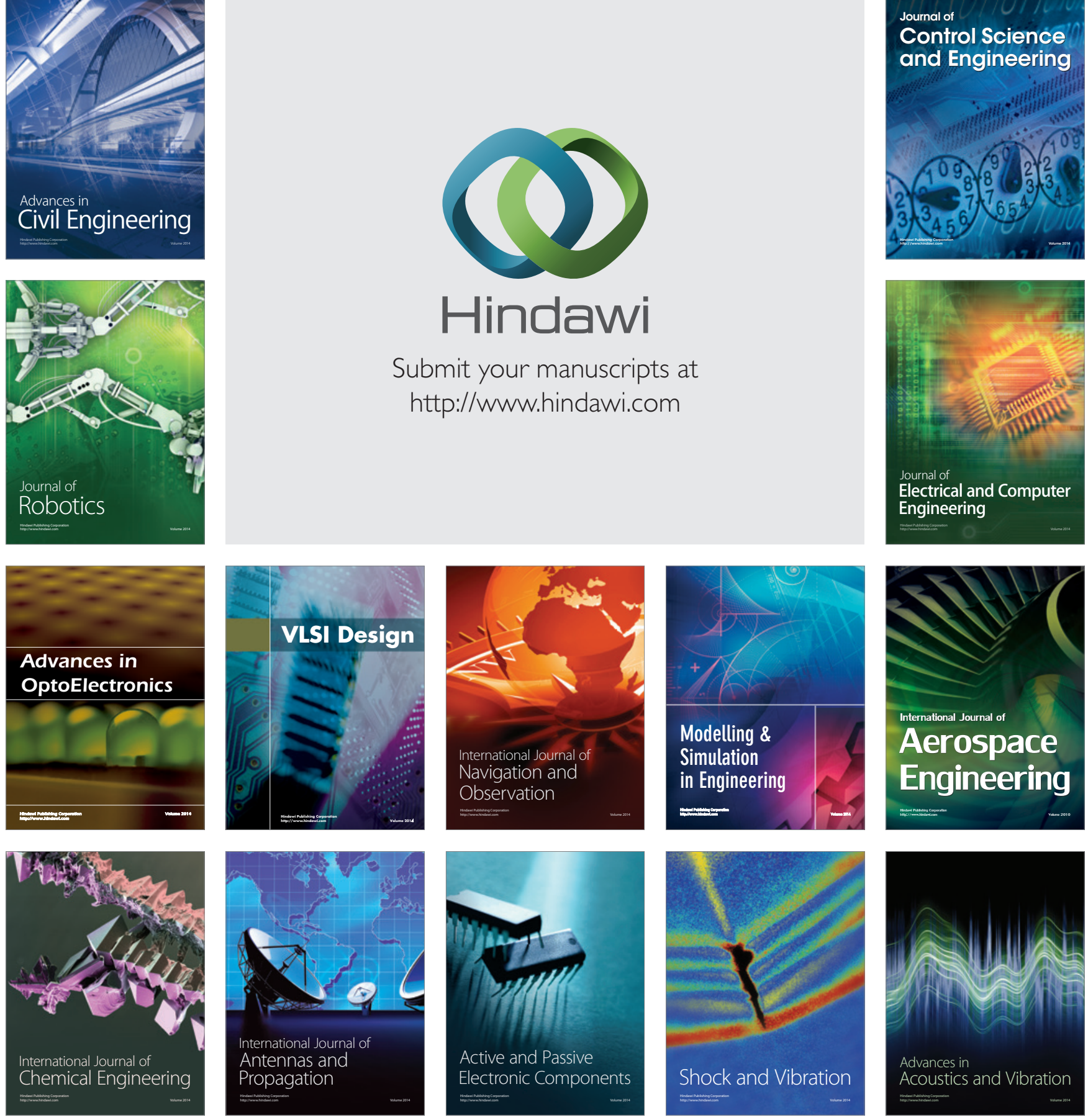